\title{
Numerical modelling and experimental validation of dynamic fracture events along weak planes
}

\author{
Irene Arias ${ }^{\text {b,* }}$, Jaroslaw Knap ${ }^{\text {c }}$, Vijaya B. Chalivendra ${ }^{\text {d }}$, Soonsung Hong a , \\ Michael Ortiz ${ }^{\mathrm{a}}$, Ares J. Rosakis ${ }^{\mathrm{a}}$ \\ a Graduate Aeronautical Laboratories, California Institute of Technology, Pasadena, CA 91125, USA \\ ${ }^{\mathrm{b}}$ Dep. de Matemàtica Aplicada III, Universitat Politècnica de Catalunya, Barcelona 08034, Spain \\ ${ }^{\mathrm{c}}$ Lawrence Livermore National Laboratory, CA 94550, USA \\ ${ }^{\mathrm{d}}$ Department of Mechanical Engineering, University of Massachusetts, North Dartmouth, MA 02747, USA
}

Received 1 November 2005; accepted 30 October 2006

Available online 31 March 2007

\begin{abstract}
The conceptual simplicity and the ability of cohesive finite element models to describe complex fracture phenomena makes them often the approach of choice to study dynamic fracture. These models have proven to reproduce some experimental features, but to this point, no systematic study has validated their predictive ability; the difficulty in producing a sufficiently complete experimental record, and the intensive computational requirements needed to obtain converged simulations are possible causes. Here, we present a systematic integrated numerical-experimental approach to the verification and validation (V\&V) of simulations of dynamic fracture along weak planes. We describe the intertwined computational and the experimental sides of the work, present the V\&V results, and extract general conclusions about this kind of integrative approach.
\end{abstract}

(C) 2007 Elsevier B.V. All rights reserved.

Keywords: Dynamic fracture; Large scale simulations; Cohesive zone model; Validation; Verification; Hopkinson bar; Photoelasticity; Inverse problem

\section{Introduction}

In the field of brittle dynamic fracture and fragmentation, the almost entirely empirical design and optimization of structural systems in ballistic and impact applications results in a very expensive case by case approach. The inability to accurately predict dynamic fracture rules out potentially advantageous manufacturing procedures. This situation is mostly due to the phenomenal computational difficulty in describing the nucleation, propagation and branching of cracks with possibly complex topologies, and to effectively resolve the huge span of scales involved, from the sample size to the small-scale physical processes at the crack tip. A number of computational techniques have

\footnotetext{
* Corresponding author. Tel.: +3493 401 1072; fax: +34 93401182. E-mail address: irene.arias@upc.edu (I. Arias).
}

been proposed, which manage to qualitatively reproduce experimental observations. In particular, finite element implementations of cohesive-zone models have been widely used to simulate fracture processes for various materials, including polymers [26], metallic materials [32], ceramic materials [5], bimaterial systems in polymer matrix composites [35], metal matrix composites [10], fiber reinforced plastic composites $[9,16]$, concrete [29], functionally graded materials [37], and ferroelectrics [2]. These models have also been used to simulate fracture under static $[21,34,14]$, dynamic $[35,5,29,30,11,37]$, and cyclic $[36,31,2]$ loading conditions. Nevertheless, agreement between simulation and experiment is achieved only after a careful casespecific parameter fitting, and thus the present methods are devoid of real predictive ability. For instance, Hao et al. [11] recently validated simulations of intersonic crack growth along weak planes under asymmetric dynamic loading, which was experimentally identified by Rosakis 
et al. $[28,27]$. In their simulations, due to the nature of the experiments, they had to guess the loading conditions from indirect experimental data. These experiences manifest the need for a simulation/validation-driven experimental design.

We report on recent efforts to develop an integrated experiment/simulation approach to the verification and validation of massively parallel finite element dynamic fracture simulations using cohesive elements. More specifically, we address here the case of interfacial fracture. The inspiring guidelines in this effort are (1) an integrated design of the experiments and the simulations with the premise of avoiding unnecessary challenges to the simulation while probing the essential features, (2) direct one-to-one comparison based on a set of relevant predefined metrics, (3) avoidance of case-specific parameter-fitting to test for predictive ability, (4) reduction of uncertainty levels through independent experimental parameter determination (e.g. loading conditions and cohesive law). A block diagram of the integrated approach used in this study is shown in Fig. 1. Key aspects in carrying out this program include on the experimental side high-resolution full-field diagnostics, and well controlled and well instrumented reproduc-

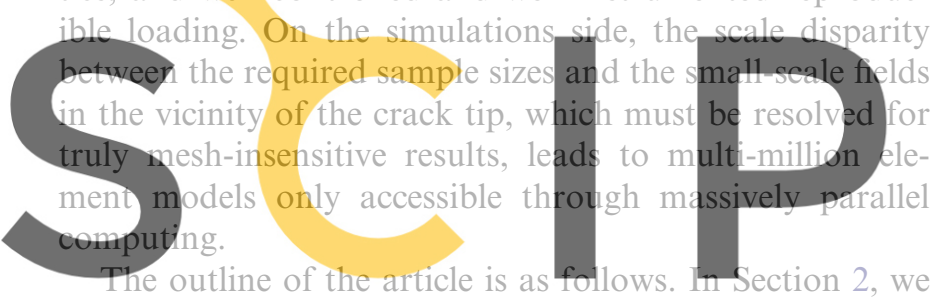
describe the cohesive finite element model of dynamic frac-

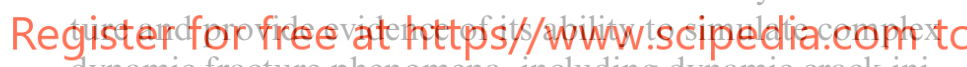
dynamic fracture phenomena, including dynamic crack initiation, crack propagation following arbitrary paths, and hierarchical branching. The design considerations from the integrated $\mathrm{V} \& \mathrm{~V}$ program and the resulting $\mathrm{V} \& \mathrm{~V}$ test are presented in Section 3. Both the experimental configuration and the simulation scheme are presented and justified thoroughly. In Section 4, results of the V\&V test are presented and discussed. We sum up and close in Section 5.

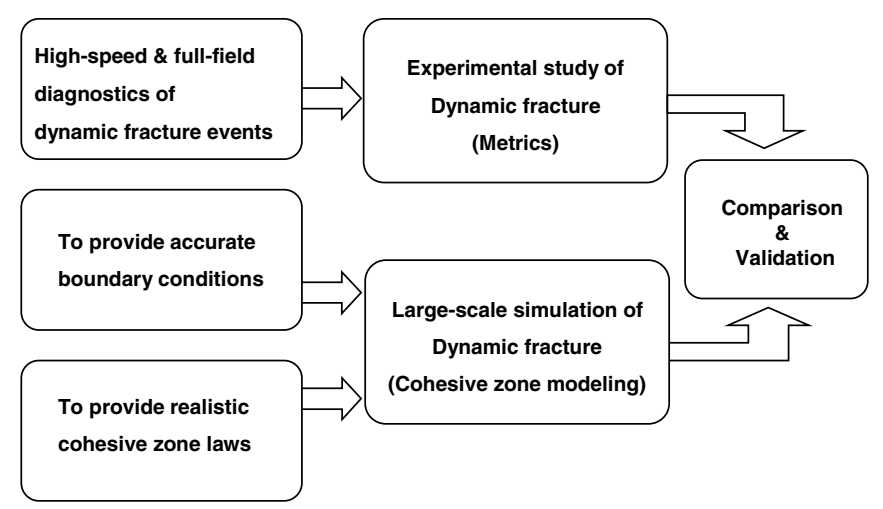

Fig. 1. A block diagram of the integrated approach for the validation of large-scale simulations of dynamic fracture.

\section{The cohesive finite element model for dynamic fracture}

Explicit treatments of fracture surfaces in finite element approaches have gained popularity in recent years. In the most straightforward and effective approach, cohesive theories of fracture dating back to Dugdale [8] and Barenblatt [3] are introduced through cohesive elements embedded in the bulk discretization [5,35]. This allows to decouple the bulk constitutive behavior and the fracture characteristics of the material. These cohesive elements bridge nascent surfaces of decohesion and govern their separation through an irreversible cohesive law, which encodes the microscopical physical processes near the crack tip. The parameters defining the cohesive law can be measured from experiments, and can also be derived from first principles. This conceptually simple technique has been proven to be very powerful in dealing with challenging issues, such as crack nucleation and branching, in a natural manner. It also provides a simple means of incorporating additional physics into the description of separation processes, including friction after debonding, chemistry, corrosion, closure, and hysteresis to name a salient few. Its simplicity makes it amenable to massively parallel simulations. Its main draw-

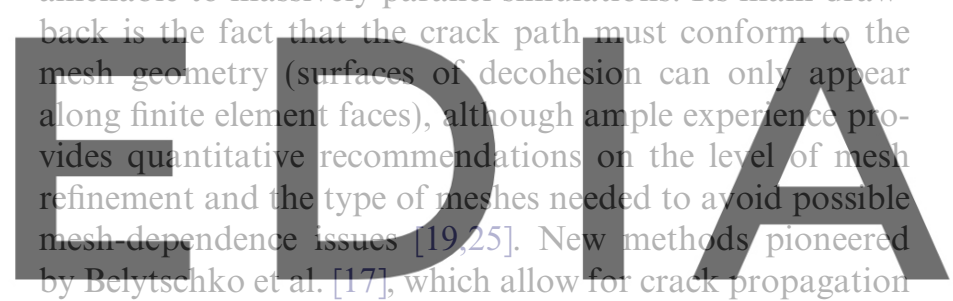

independently of the mesh, have strongly emerged in recent

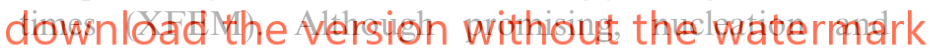
branching are still issues to be fully addressed in these approaches, despite recent efforts to use damage models in these respects for the case of quasi-static fracture [1]. On the other hand, their algorithmic complexity poses doubts on their implementation in large scale calculations.

The present implementation of finite deformation cohesive elements is due to Camacho and Ortiz [5]. As described in [23], the model is greatly simplified by the additional assumption that the cohesive law derives from a cohesive potential that depends on a scalar effective opening displacement, which is a weighted norm of the normal and tangential components, thus differentiating between mode I and modes II and III of fracture. In addition, the initially rigid cohesive elements are adaptively inserted as needed, as indicated by the fracture criterion induced by the cohesive law, in the form of

$t=\sqrt{\beta^{-2}\left|\mathbf{t}_{S}\right|+t_{n}^{2}} \leqslant t_{\max }, \quad t_{\max }=\alpha \sigma_{c}$

where $t_{n}$ and $\left|\mathbf{t}_{S}\right|$ are the normal and tangential components of the traction vector at an interface between two elements; $\sigma_{c}$ is the fracture strength of the material and $\alpha$ is a parameter. Each cohesive element insertion introduces major topological changes in the vicinity of the two elements. These changes involve insertion of such topological entities 

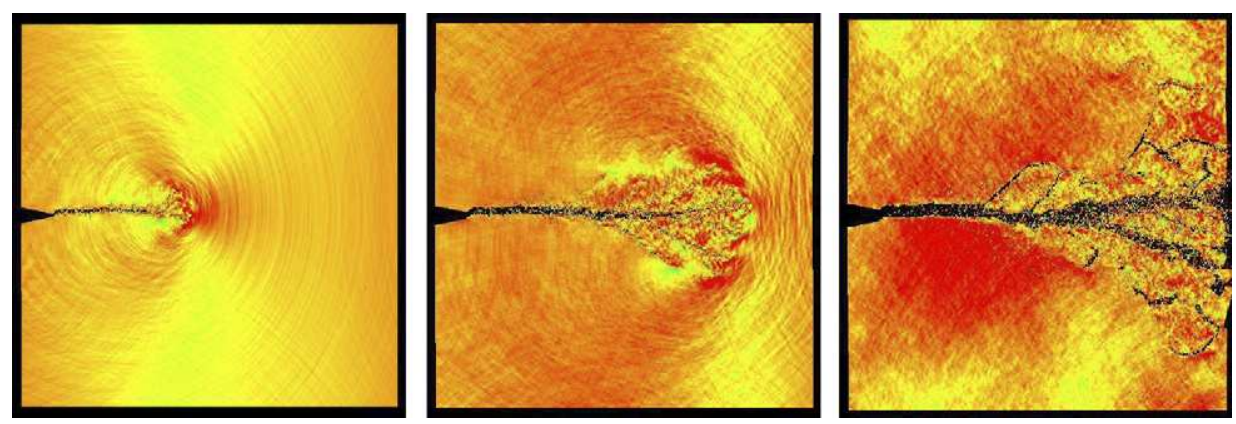

Fig. 2. Snapshots (increasing times to the right) of the fracture process of a square pre-notched PMMA plate subjected to an initial uniform tensile strain rate. Colors indicate level of a relevant stress. (For interpretation of colour representation in this figure legend, the reader is referred to the web version of this article.)

as nodes, edges or faces, which, in turn, leads to the formation of free surfaces between some of the volume elements. Coalescence of these newly formed cracks may lead eventually to the formation of new bodies (fragmentation). For a detailed description of the parallel fracture and fragmentation algorithm see [15].

The versatility and ability to describe complex fracture processes of cohesive finite elements have been demonstrated in numerous works $[21,35,5,23,24,29,30,22]$. Next,

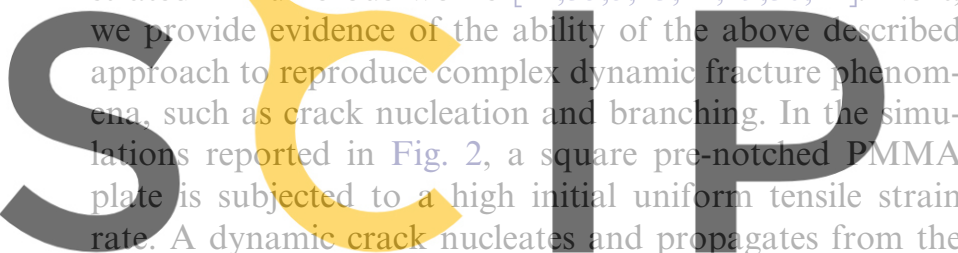
notch, and as sufficiently large energy input is provided, Register for free at httos the point in which. it becomes fragmentation. The characteristic hierarchical crack pattern is apparent in the later snapshots. The ability to qualitatively reproduce a myriad of other dynamics cracking events has also been investigated, such as the crack deflection/penetration behavior of cracks approaching inclined interfaces [33]. Nevertheless, the question remains about the real predictive ability of this kind of simulations, beyond realistic simulation results.

To address this question, we follow a validation strategy based on a divide-and-conquer scheme. Instead of attempting the validation of the ability of the model to nucleate new cracks, to propagate cracks through the specimen or to describe branching in a single experiment, we rather focus on individual fracture events, understood as unit processes. The first step, described here, focuses on the crack propagation along weak planes. Other fracture events leading to more complex crack patterns are currently being addressed and will be reported elsewhere.

\section{Design considerations for the validation test}

The previously described integrated approach is based on a unified design of the validation tests as a whole. Experiments and simulations place restrictions on each other, which must not seriously impair the feasibility and simplicity of the overall V\&V test. We avoid the practice of trying to reproduce data from the literature but rather design experiments specifically targeted to the validation effort. These experiments, while probing the fundamental fracture processes, must be simple and reduce unproductive challenges to the numerical model.

The design that has emerged from this process is described and justified next. A simple specimen configura-

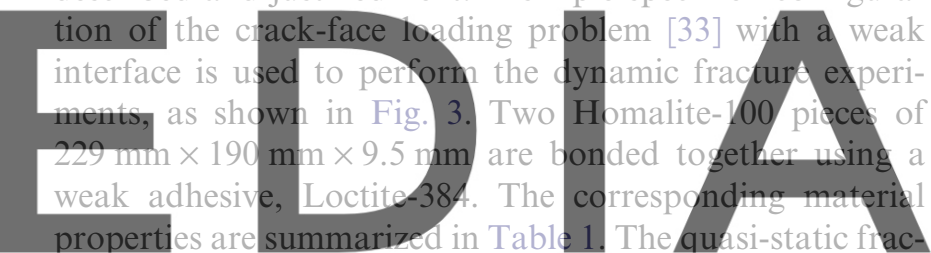
ture toughness of the Loctite-384 adhesive when used to bond Homalite-100 sheets is $0.35 \mathrm{MPa} \mathrm{m}^{1 / 2}$. A small notch implemented numerically by an interface of cohesive elements with the cohesive properties of the glue. Dynamic photoelasticity in conjunction with high-speed photography is used to capture real-time records of the crack initiation

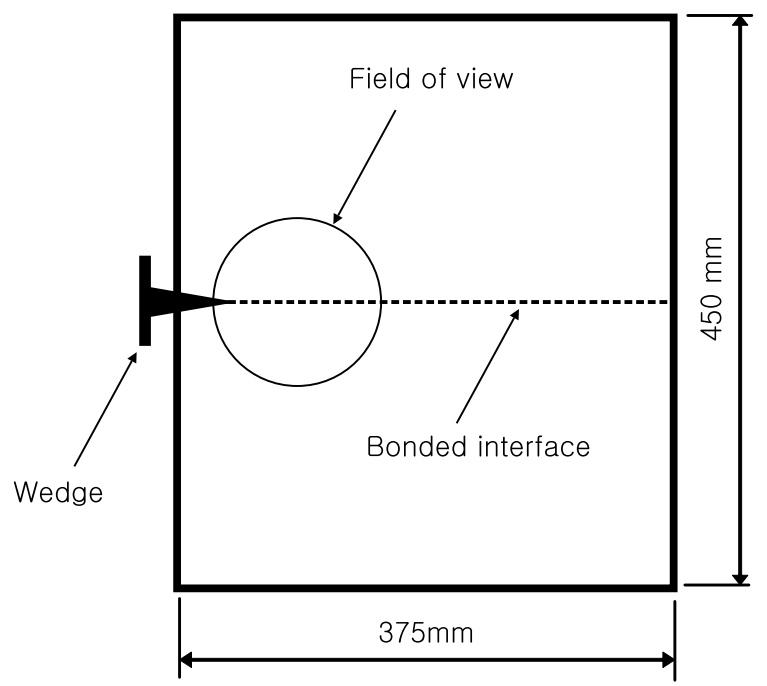

Fig. 3. A specimen configuration of the dynamic fracture experiment along a weak interface. 
Table 1

Material properties of Homalite-100

\begin{tabular}{lll}
\hline Property & $\begin{array}{l}\text { Static } \\
\left(\dot{\varepsilon}=10^{-3} / \mathrm{s}\right)\end{array}$ & $\begin{array}{l}\text { Dynamic } \\
\left(\dot{\varepsilon}=10^{3} / \mathrm{s}\right)\end{array}$ \\
\hline Density $\left(\mathrm{kg} / \mathrm{m}^{3}\right)$ & 1230 & 1230 \\
Young's modulus $(\mathrm{GPa})$ & 3.45 & 4.8 \\
Dilatational wave speed $(\mathrm{m} / \mathrm{s})$ & 1890 & 2119 \\
Shear wave speed $(\mathrm{m} / \mathrm{s})$ & 1080 & 1208 \\
Rayleigh wave speed $(\mathrm{m} / \mathrm{s})$ & 1010 & 1110 \\
Poisson's ratio & 0.35 & 0.35 \\
\hline
\end{tabular}

and propagation during the dynamic fracture experiment. A typical experimental record is shown in Fig. 4. For additional experimental details, see [6].

For the purpose of validating the fracture models, it is essential to separate the bulk constitutive behavior from the fracture response. For this reason, brittle materials are employed in the tests. Another requirement that the validation effort imposes on the material is the need for full-field diagnostics. The photo-elastic and brittle characteristics of Homalite-100 make of this material an ideal choice. Homalite-100 is a brittle polyester resin whose mechanical response is mildly rate sensitive [27]. This brittle material is modelled using hyper-elasticity (the deformations are small but the displacements and rotations car be
large) and the dynamic value for Young's modulus as
reported by [7]. It should be noted that, for the specific val-
idation tests described here, the cohesive properties of
Homalite-100 are irrelevant since the crack propagates along the weak plane. Furthermore, the fact that the crack path is known a priori eliminates any orientation-related mesh dependence issue. This is not the case in ongoing efforts dealing with different configurations.

For a clean validation of the fracture processes, it is important to decouple other mechanical effects such as complex wave propagation patterns. Therefore, the sample sizes must be large enough for the reflected waves from the sample boundaries not to enter the field of view during the observation time. The sample thickness allows for a twodimensional treatment without significant loss of accuracy. Indeed, as observed by [24], the cracks propagating in thin plates do not exhibit fully three-dimensional character.

The choice of Loctite-384 as the weak plane material requires that the characteristic length $l_{c}=1.267 \mathrm{~mm}$ be resolved as needed by the requirement the simulation results be independent of the details of the finite element discretization. Consequently, if one decides to treat the original problem as two-dimensional (2D), and moreover, assume the characteristic length be spanned by +5 elements, the overall number of elements in a triangular discretization of the experimental sample may easily exceed +5 millions. Furthermore, the time records needed for val-

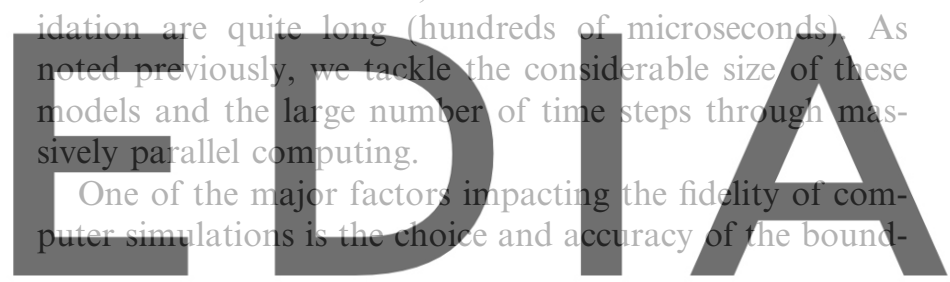

Register for free at littps//www.scipedia.com to blownload the version without the watermark
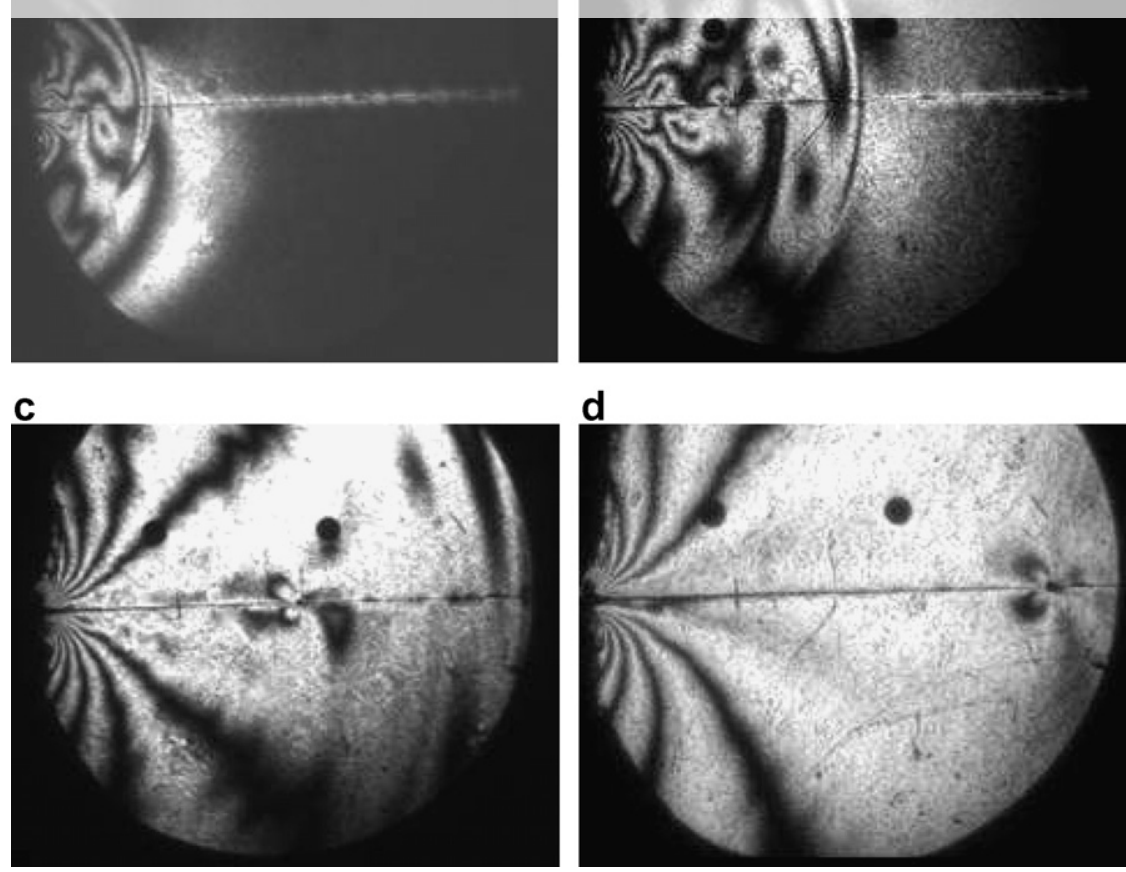

d

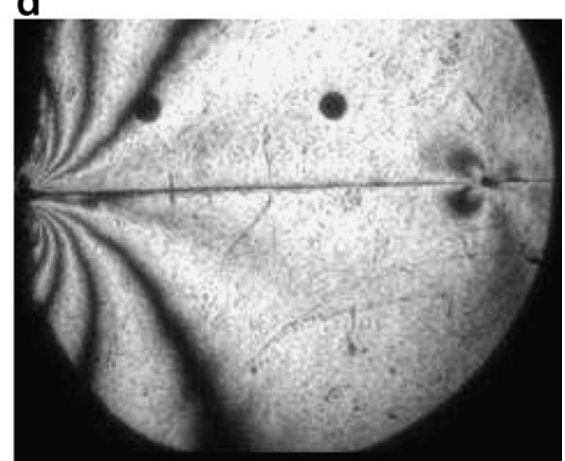

Fig. 4. Photoelastic fringes (contours of maximum shear stress) of a dynamic crack propagation along a weak interface as captured by the high-speed camera. (a) $t=18 \mu \mathrm{s}, a=12.1 \mathrm{~mm}$; (b) $t=48 \mu \mathrm{s}, a=32.7 \mathrm{~mm}$; (c) $t=93 \mu \mathrm{s}, a=65.3 \mathrm{~mm}$ and (d) $t=153 \mu \mathrm{s}, a=110.0 \mathrm{~mm}$. 
ary conditions. Complex boundary conditions are not easily amenable to computer simulations, and may severely degrade the accuracy of computer predictions. Moreover, the translation from the experimentally available data on the loading to the actual loading on the numerical model often requires a modelling step on the loading device. Our experimental set-up and instrumentation have been designed to eliminate any uncertainties regarding the boundary conditions (c.f. Fig. 5). As the sample is not constrained in any way once impacted, we model the sample as traction free. The crack notch vicinity and the loading device (a modified Hopkinson bar) have been instrumented with strain gages for a direct measurement of the actual loading pulse (c.f. [6] for details). The recorded data is supplied as input to the simulation. This approach has proven very effective and allowed the boundary conditions to remain in strict agreement with their experimental counterparts.

There is a common assumption in the literature on cohesive finite element simulations of fracture that details on the cohesive law beyond two independent parameters (the cohesive energy, and either of the cohesive strength or the separation length) do not affect significantly the results.

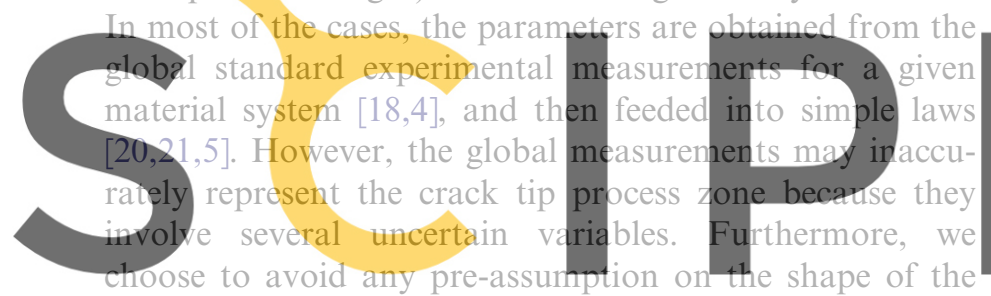
cohesive law and the importance of this shape. The novelty

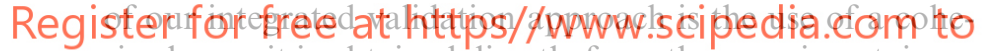
sive law as it is obtained directly from the experiment, in an effort to reduce unnecessary uncertainties. A framework developed for the inverse analysis of the crack tip cohesive response from measurements of the crack tip fields is employed [12,13]. This approach combines experimental, numerical and analytical methods to obtain a realistic cohesive law of the weak interface. The resulting cohesive law is shown in Fig. 6.

The choice of metrics is at the heart of any V\&V effort. These metrics should be fundamental to the underlying

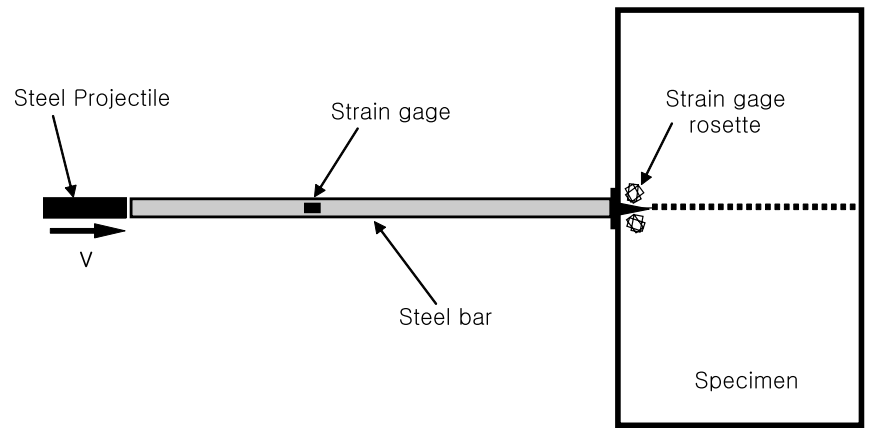

Fig. 5. An experimental setup using a modified Hopkinson bar for wellcontrolled loading boundary conditions of dynamic crack-face loading problem.

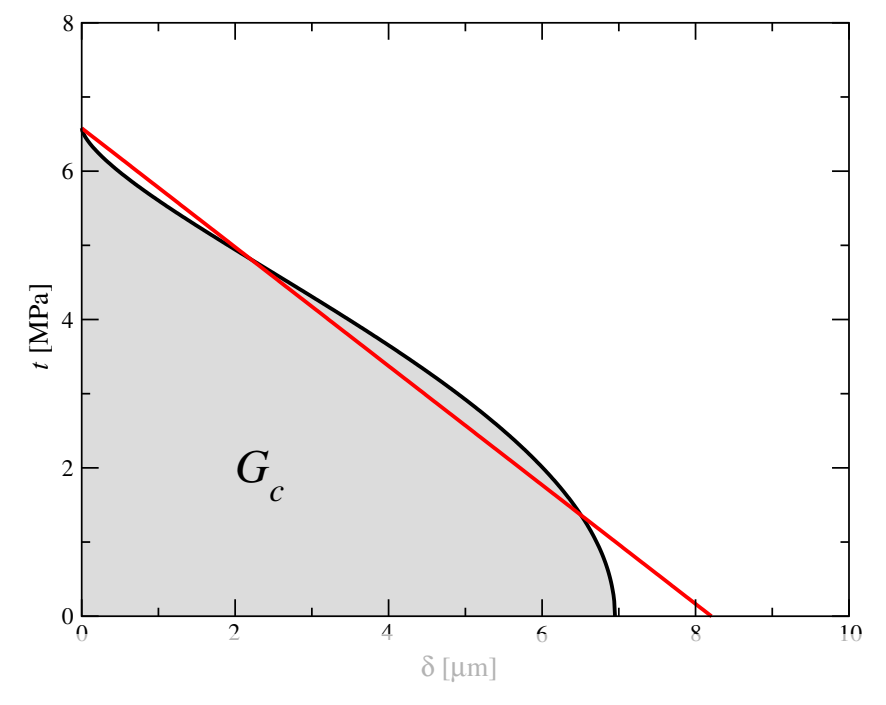

Fig. 6. Experimentally obtained cohesive law and corresponding linear cohesive law.

phenomena and be amenable to accurate experimental instrumentation. Moreover, they should be easily extractable from simulation results. Here, we employ the follow-

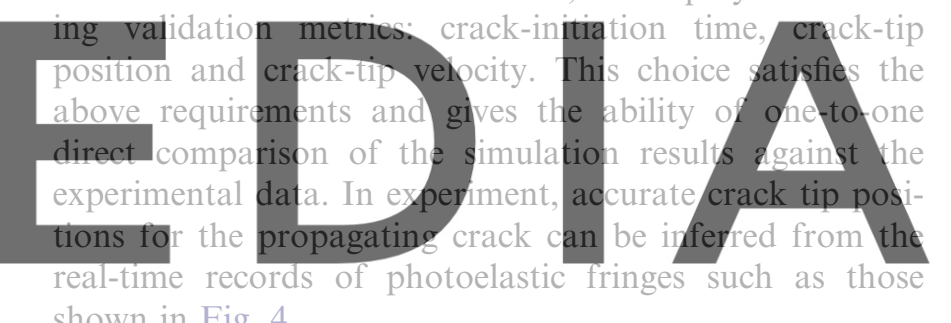
shown in Fig. 4.

\section{download the version without the watermark}

\section{Verification and validation results}

The results of the above described $\mathrm{V} \& \mathrm{~V}$ program are presented next. Verification results include (1) an analysis of convergence to grasp on the effect of under-resolution, and (2) a sensitivity analysis to investigate the effect of the shape of the cohesive law on the simulated fracture response. The validation results include the results of the direct one-to-one comparisons between experimental and numerical records of the predefined metrics.

\subsection{Convergence analysis}

As a result of the design considerations described in Section 3, we employ large uniform triangular finite element meshes and resort to massively parallel computing to tackle the complexity of the problem. Ample experience provides quantitative recommendations on the level of mesh refinement needed for mesh-insensitive results. Nevertheless, we analyze the convergence properties of our numerical model, for a precise quantification of the required level of resolution. Five different mesh sizes are considered. The coarser mesh has an element size of $h=5.27 \mathrm{~mm}$, corresponding to a value of $h / l_{c}=4.16, l_{c}=1.267 \mathrm{~mm}$ being 
the characteristic cohesive length of Loctite-384. Finer meshes are obtained from the coarse mesh by recursive uniform subdivision. The finer mesh has an element size of $h=0.33 \mathrm{~mm}$, or $h / l_{c}=0.26$ (i.e. approximately four elements per the characteristic cohesive length). Fig. 7 shows the computed position of the crack tip for the different mesh sizes. As expected, for coarse meshes, the predictions of the model depend strongly on the element size $h$. For values of $h$ smaller than $l_{c}$, the numerical predictions converge: the curves corresponding to $h / l_{c}=0.54$ and $h / l_{c}=0.26$ are indistinguishable. It is noteworthy that here convergence refers to the mesh size only, since the crack path is known a priori.

\subsection{Sensitivity to cohesive law}

As mentioned previously, there is the common knowledge in cohesive finite element models of brittle fracture

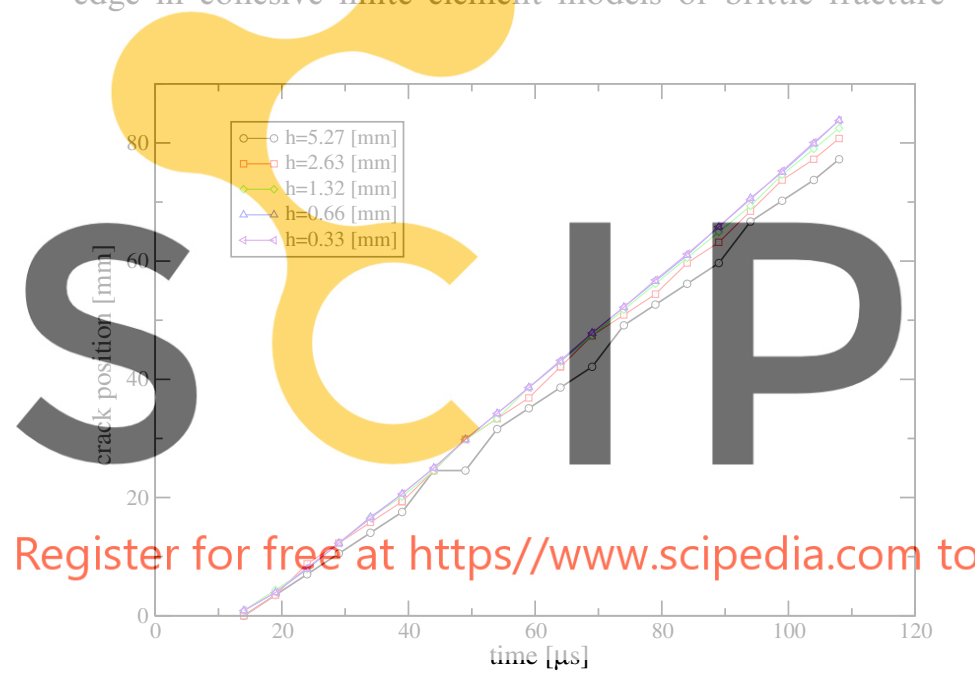

Fig. 7. Crack tip position vs. time obtained in the simulation for uniform finite element meshes of different size.

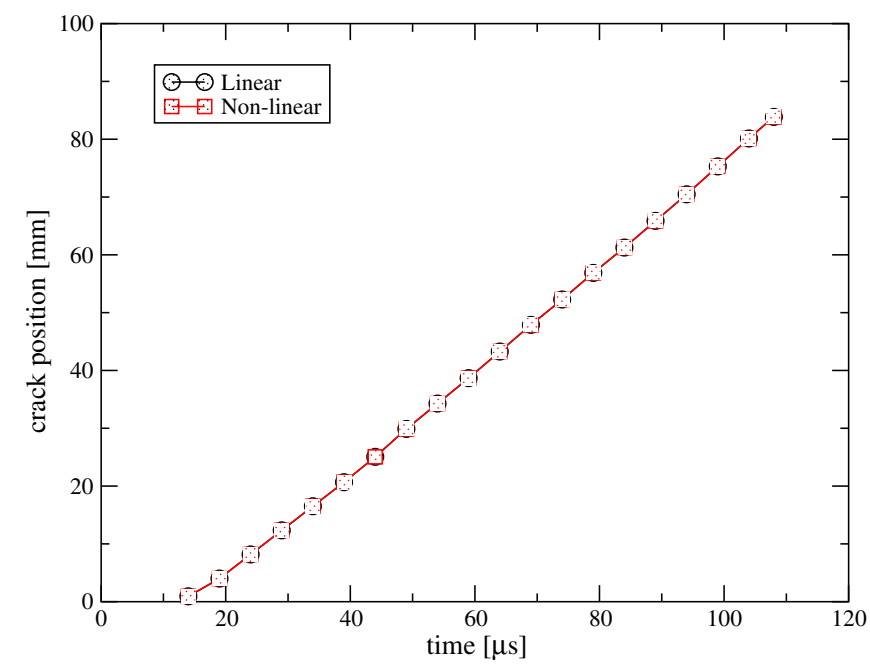

Fig. 8. Crack tip vs. time for Experiment 1 with two different cohesive laws. that the functional shape of the cohesive law does not have a significant effect on the fracture response. Thus, the most popular and extensively used cohesive laws in the literature (e. g., $[20,21,5])$ depend only on two parameters (the cohe-
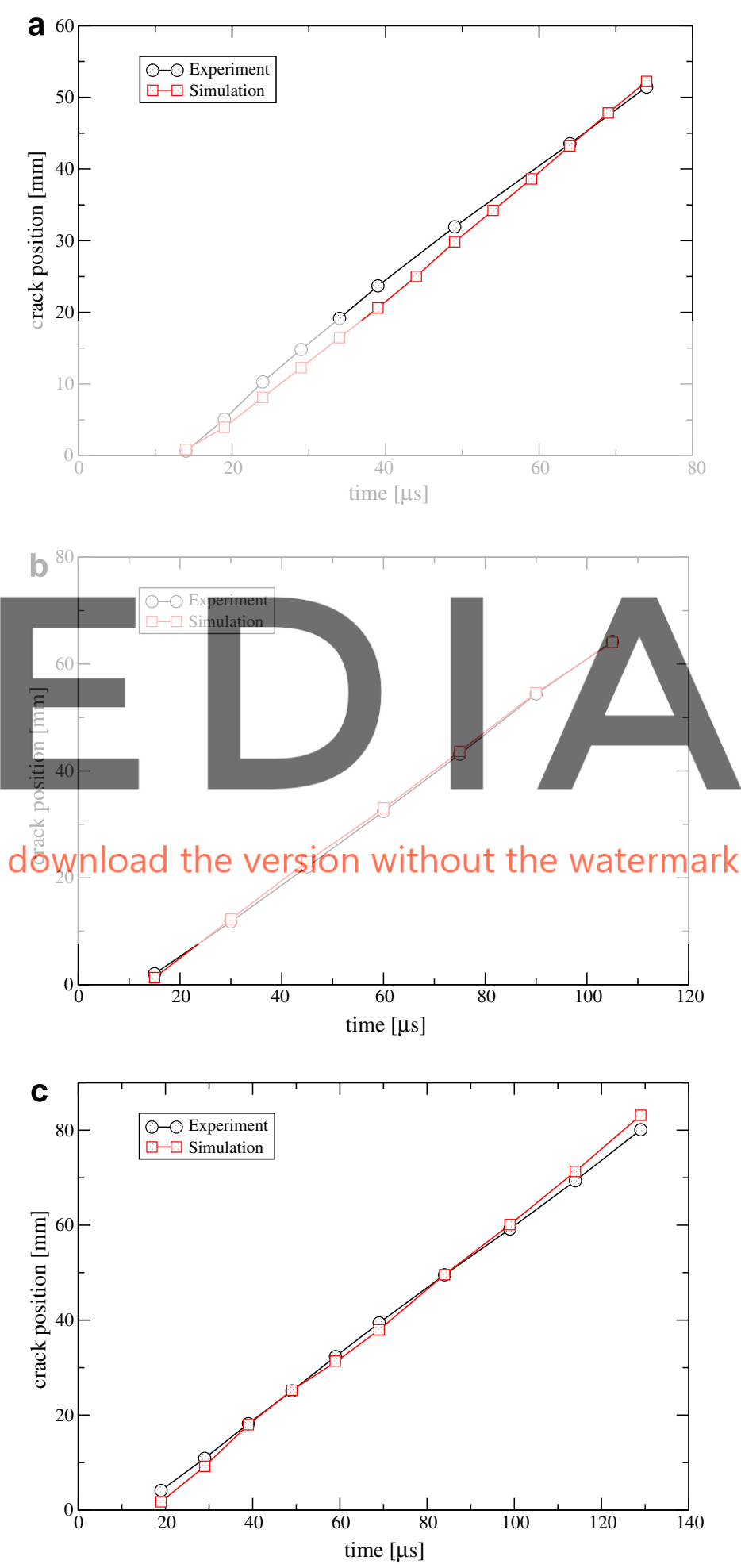

Fig. 9. Crack tip position vs. time; black curve - experiment, red curve simulation. (a) Experiment 1, (b) experiment 2 and (c) experiment 3. (For interpretation of colour representation in this figure legend, the reader is referred to the web version of this article.) 
Table 2

Crack tip velocity for Experiment 1, 2 and 3

\begin{tabular}{llll}
\hline \multirow{2}{*}{ Experiment no. } & \multicolumn{2}{l}{ Crack tip velocity $[\mathrm{m} / \mathrm{s}]$} & Relative error [\%] \\
\cline { 2 - 3 } & Experiment & Simulation & \\
\hline 1 & 844 & 870 & 3.1 \\
2 & 697 & 698 & 0.1 \\
3 & 688 & 729 & 6.0 \\
\hline
\end{tabular}

sive energy, and either of the cohesive strength or the separation length). We test here this assumption in the present configuration by comparing the model predictions obtained with two different cohesive laws: one is the experimentally obtained cohesive law; the other is a linear cohesive law with the same two relevant parameters, namely the fracture energy and the critical traction. The crack tip position computed with each of these cohesive laws is plotted in Fig. 8 as a function of time. It is apparent that the results for the present configuration do not depend on the shape of the cohesive law.

4.3. Validation results

Three different experimental realizations have been con

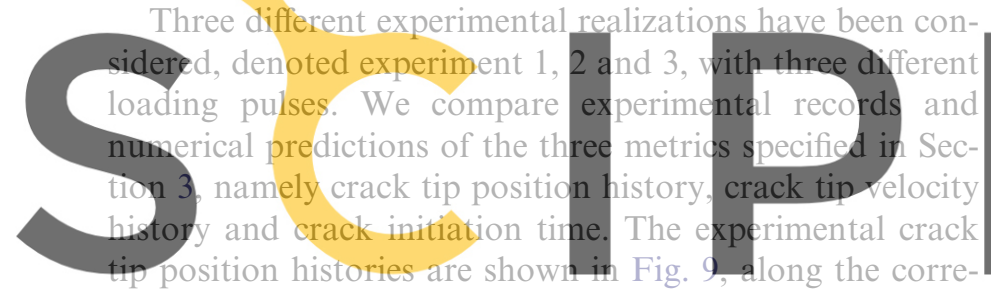
sponding model predictions. The agreement between exper-

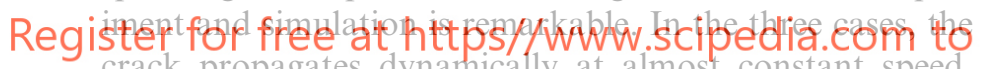
crack propagates dynamically at almost constant speed tories are obtained from these curves as the slope of the linear regression fit to the data and presented in Table 2, along with the relative error of the numerical predictions with respect to the experimental data. All three relative errors are below $6 \%$, the lowest being $0.1 \%$ in the case of Experiment 2. Finally, it is apparent that in all three experiments, the crack starts propagating dynamically at $13 \mu \mathrm{s}$, exactly the same time predicted by the model within experimental resolution.

\section{Summary and concluding remarks}

Despite cohesive finite element models have demonstrated their ability to simulate complex dynamic fracture phenomena, in particular dynamic crack initiation, crack propagation following arbitrary paths, and hierarchical branching, no systematic V\&V approach has been attempted to assess their fidelity. We have addressed this challenge by designing an integrated experiment/simulation approach to the verification and validation of massively parallel cohesive finite element dynamic fracture simulations. We have presented an application to the case of dynamic fracture along weak planes. The inspiring guidelines in this effort are (1) an integrated design of the experiments and the simulations with the premise of avoiding unnecessary challenges to the simulation while probing the essential features, (2) direct one-to-one comparison based on a set of relevant predefined metrics, (3) avoidance of case-specific parameter-fitting to test for predictive ability, (4) reduction of uncertainty levels through independent experimental parameter determination (e.g. loading conditions and cohesive law).

The validation test is designed as a whole by accounting for the often conflicting requirements that experiment and numerical simulation place on each other. In a divide-andconquer strategy, we focus the validation test on the specific fracture event (unit process): dynamic crack propagation along weak planes. The validation test specifications derive from the following design considerations: (1) a brittle material to decouple fracture from constitutive response, (2) a photoelastic material to allow for real-time, full-field diagnostics based on dynamic photoelasticity in conjunction with high-speed photography, (3) a large enough sample to isolate fracture response in the field-ofview during the observation time from the elastic wave reflections from the sample boundaries, (4) a thin plate

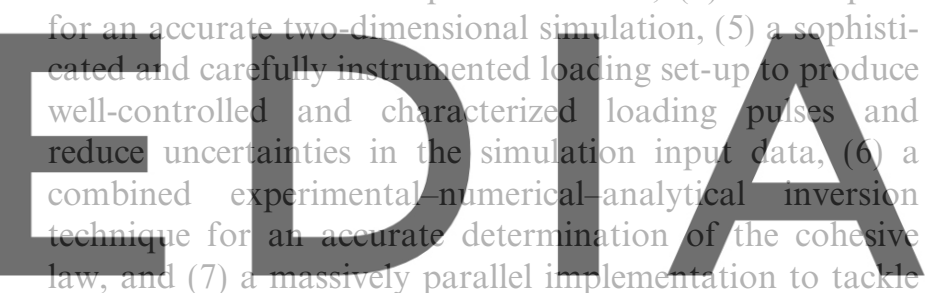
the huge complexity of the resulting finite element model.

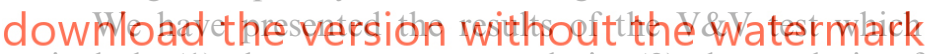
include (1) the convergence analysis, (2) the analysis of the sensitivity of the model predictions to the functional shape of the cohesive law revealing no significant effect of the shape of the cohesive law for the present configuration, and (3) the excellent agreement in direct one-to-one comparisons of the experimental and the simulated records of the selected relevant metrics: crack tip position history, crack velocity history and crack initiation time. A perfect agreement of the latter is outstanding.

\section{Acknowledgements}

We gratefully acknowledge support from the US Department of Energy through Caltech's ASC Center for the Simulation of Dynamic Response of Materials, from the European Commission through grant IRG FP6029158 to IA, and from the Barcelona Supercomputing Center - Centro Nacional de Supercomputación.

\section{References}

[1] P.M.A. Areias, T. Belytschko, Analysis of three-dimensional crack initiation and propagation using the extended finite element method, Int. J. Numer. Meth. Engrg. 63 (5) (2005) 760-788. 
[2] I. Arias, S. Serebrinsky, M. Ortiz, A phenomenological cohesive model of ferroelectric fatigue, Acta Mater. 54 (4) (2006) 975-984.

[3] G.I. Barenblatt, The mathematical theory of equilibrium cracks in brittle fracture, Adv. Appl. Mech. 7 (1962) 55-129.

[4] T.W. Bjerke, J. Lambros, Theoretical development and experimental validation of a thermally dissipative cohesive zone model for dynamic fracture of amorphous polymers, J. Mech. Phys. Solids 51 (6) (2003) $1147-1170$.

[5] G.T. Camacho, M. Ortiz, Computational modelling of impact damage in brittle materials, Int. J. Numer. Meth. Engrg. 33 (20-22) (1996) 2899-2938.

[6] V.B. Chalivendra, S. Hong, J. Knap, I. Arias, M. Ortiz, J.R.A, Experimental validation of large scale simulations of dynamic fracture along weak planes. Submitted, 2005.

[7] J.W. Dally, A. Shukla, Energy loss in homalite 100 during crack propagation and arrest, Engrg. Fract. Mech. 13 (4) (1980) 807-817.

[8] D.S. Dugdale, Yielding of steel sheets containing slits, J. Mech. Phys. Solids 8 (2) (1960) 100-104.

[9] H.D. Espinosa, S. Dwivedi, H.C. Lu, Modeling impact induced delamination of woven fiber reinforced composites with contact/ cohesive laws, Comp. Methods Appl. Mech. Engrg. 183 (3-4) (2000) 259-290.

[10] J.W. Foulk, D.H. Allen, K.L.E. Helms, Formulation of a threedimensional cohesive zone model for application to a finite element algorithm, Comp. Methods Appl. Mech. Engrg. 183 (1-2) (2000) $51-66$.

[11] S. Hao, W.K. Liu, P.A. Klein, A.J. Rosakis, Modeling and simulation of intersonic crack growth, Int. J. Sol. Struct. 41 (7) (2004) 1773-1799.

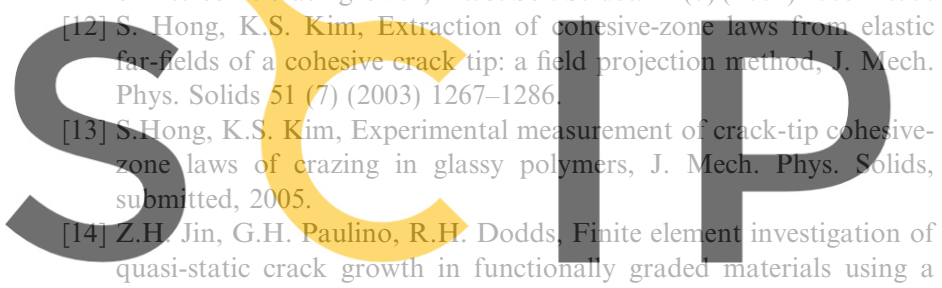
novel cohesive zone fracture model, J. Appl. Mech. - Trans. ASME

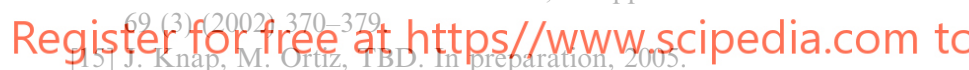

[16] S. Li, M.D. Thouless, A.M. Waas, J.A. Schroeder, P.D. Zavattieri, Use of a cohesive-zone model to analyze the fracture of a fiberreinforced polymer-matrix composite, Compos. Sci. Technol. 65 (3-4) (2005) 537-549.

[17] N. Moës, J. Dolbow, T. Belytschko, A finite element method for crack growth without remeshing, Int. J. Numer. Meth. Engrg. 46 (1) (1999) 131-150.

[18] I. Mohammed, K.M. Liechti, Cohesive zone modeling of crack nucleation at bimaterial corners, J. Mech. Phys. Solids 48 (4) (2000) $735-764$.

[19] J.F. Molinari, G. Gazonas, R. Raghupathy, A. Rusinek, F. Zhou, The cohesive element approach to dynamic fragmentation: The question of energy convergence, Int. J. Numer. Meth. Eng. In Press, 2006.

[20] A. Needleman, A continuum model for void nucleation by inclusion debonding, J. Appl. Mech. - Trans. ASME 54 (3) (1987) 525-531.

[21] A. Needleman, An analysis of decohesion along an imperfect interface, Int. J. Fract. 42 (1) (1990) 21-40.

[22] O. Nguyen, E.A. Repetto, M. Ortiz, R.A. Radovitzky, A cohesive model of fatigue crack growth, Int. J. Fract. 110 (4) (2001) 351369.

[23] M. Ortiz, A. Pandolfi, Finite-deformation irreversible cohesive elements for three-dimensional crack-propagation analysis, Int. J. Numer. Meth. Engrg. 44 (9) (1999) 1267-1282.

[24] A. Pandolfi, P.R. Guduru, M. Ortiz, A.J. Rosakis, Three dimensional cohesive-element analysis and experiments of dynamic fracture in c300 steel, Int. J. Sol. Struct. 37 (27) (2000) 3733-3760.

[25] K.D. Papoulia, S.A. Vavasis, P. Ganguly, Spatial convergence of crack nucleation using a cohesive finite-element model on a pinwheelbased mesh, Int. J. Numer. Meth. Engrg. 67 (2006) 1-16.

[26] P. Rahulkumar, A. Jagota, S.J. Bennison, S. Saigal, Cohesive element modeling of viscoelastic fracture: application to peel testing of polymers, Int. J. Sol. Struct. 37 (13) (2000) 1873-1897.

[27] A.J. Rosakis, Intersonic shear cracks and fault ruptures, Adv. Phys. 51 (4) (2002) 1189-1257.

[28] A.J. Rosakis, O. Samudrala, D. Coker, Cracks faster than the shear wave speed, Science 284 (5418) (1999) 1337-1340.

[29] G. Ruiz, M. Ortiz, A. Pandolfi, Three-dimensional finite-element simulation of the dynamic brazilian tests on concrete cylinders, Int. J. Numer. Meth. Engrg. 48 (7) (2000) 963-994.
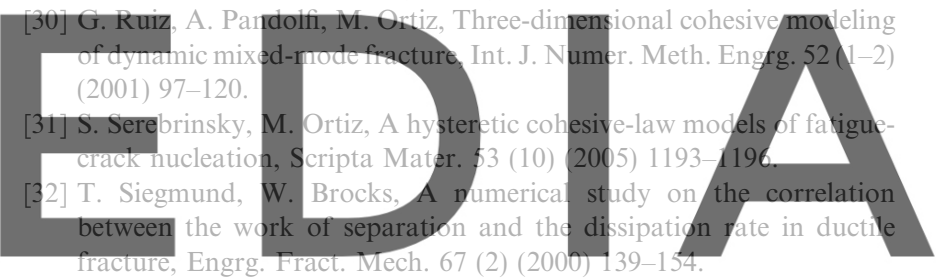

[337 L.R. Xu, Y.G.Y. Huang, A.J. Rosakis, Dynamic crack deflection and

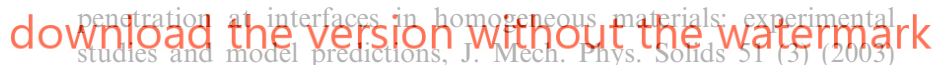
461-486.

[34] X.P. Xu, A. Needleman, Void nucleation by inclusion debonding in a crystal matrix, Model. Simulat. Mater. Sci. Engrg. 1 (2) (1993) 111132.

[35] X.P. Xu, A. Needleman, Numerical simulations of fast crack-growth in brittle solids, J. Mech. Phys. Solids 42 (9) (1994) 1397-1434.

[36] B. Yang, S. Mall, K. Ravi-Chandar, A cohesive zone model for fatigue crack growth in quasibrittle materials, Int. J. Sol. Struct. 38 (22-23) (2001) 3927-3944.

[37] Z.Y.J. Zhang, G.H. Paulino, Cohesive zone modeling of dynamic failure in homogeneous and functionally graded materials, Int. J. Plast. 21 (6) (2005) 1195-1254. 